\title{
Concepto de calidad en educación en la formación del profesional de cinesiología
}

\author{
Héctor F. Retamal-Matus, Claudia A. Estrada-Goic
}

Objetivo. Conocer el concepto de calidad educativa en la formación del profesional de cinesiología.

Sujetos y métodos. Un total de 90 participantes egresados y estudiantes de último año de la carrera de cinesiología contestaron el instrumento de evaluación que contenía los criterios de calidad en educación propuestos para la formación del profesional de cinesiología. Mediante un diseño no experimental transversal correlacional, los participantes voluntarios opinaron individualmente sobre la cuestión.

Resultados y conclusiones. Los resultados indican que, en general, todos los criterios se consideraron importantes, pero existen algunas diferencias entre profesionales y estudiantes sobre la relevancia de los criterios de calidad en educación. Los profesionales egresados de la carrera de cinesiología otorgan una mayor importancia a los criterios de calidad en educación relacionados con la excelencia y la evaluación. Además, jerarquizan como importantes criterios de calidad vinculados con los recursos, procesos y resultados. Por otro lado, los estudiantes dan gran importancia a los criterios relacionados con el proceso de formación, como son los recursos, la evaluación y la excelencia. Se discuten los resultados en torno al concepto de calidad y sus posibles aplicaciones en la mejora de los procesos educativos y, por tanto, en la formación profesional.

Palabras clave. Cinesiología. Formación profesional. Percepción de la calidad educativa. Salud.

\section{Concept of educational quality in physical therapists" undergraduate professional training}

Aim. To explore the concept of quality in education during the undergraduate years of physical therapy student's training.

Subjects and methods. A total of 90 senior students and graduated physical therapists answered the questions from a survey instrument containing the whole quality variables in education, proposed during the curriculum program of physical therapists. A non experimental transversal cross correlational study was used.

Results and conclusions. The results indicate there are significant differences among graduates and the student's perceptions about the relevance of quality components of the educational experience. The graduated therapists attributed greater importance to the areas of education related to excellence and evaluation. They also prioritized aspects of quality connected with the resources, process and outcomes. Conversely, the students gave greater importance to the areas associated with the learning process; such as the resources of the institution, assessment and better education. Outcomes related to the concept of educational quality and its possible applications in the improvement of the learning process and overall professional development are discussed.

Key words. Health. Perception of quality education. Physical therapy. Professional development.

\section{Introducción}

En la actualidad, en Chile, el concepto de calidad en educación es parte del discurso cotidiano, donde todos los actores de nuestra sociedad concuerdan en que es necesario 'mejorar la calidad' de la educación a diferente niveles. La cinesiología no escapa a estas demandas y con el objetivo de la mejora continua de la calidad en la formación de los profesionales, las carreras deben someterse a constantes procesos de evaluación y acreditación para mejorar la calidad de la educación ofrecida [1]. En este sen- tido, cabe preguntarse qué se entiende por 'calidad educativa', un concepto complejo y que entraña diversos criterios, los cuales dificultan su definición y aún más su medición.

Las primeras aproximaciones hacen referencia a una 'buena educación', a una 'buena facultad', a un 'buen plan de estudios' o a una 'buena universidad', donde el concepto se utiliza sin evidencias contrastadas [2].

Algunos autores refieren que ha llegado a ser un concepto vacío y que no existe como tal [3]. ZuritaChávez [4] sostiene que la calidad de la educación
Departamento de Kinesiología (H.F. Retamal-Matus). Departamento de Psicología (C.A. Estrada-Goic). Facultad de Humanidades, Ciencias Sociales y de la Salud. Universidad de Magallanes. Punta Arenas, Chile.

Correspondencia: Prof. Héctor Felipe Retamal Matus. Facultad de Humanidades, Ciencias Sociales y de la Salud. Universidad de Magallanes. Avda. Bulnes, 01855. CP 6200000 Punta Arenas, Chile.

E-mail:

felipe.retamal@umag.cl

Conflicto de intereses: No declarado.

Competing interests: None declared.

(c) 2014 FEM 
se ha llegado a convertir en un 'lema educacional'. Sin embargo, estos primeros acercamientos no sirven para aclarar el problema de la definición y de cómo se mide la calidad en educación. Por ello, la UNESCO menciona que la calidad es un valor que requiere definirse en cada situación, que no puede entenderse como un valor absoluto y que los significados que se atribuyan a la calidad de la educación dependerá de algunos aspectos como la perspectiva social de los sujetos que la definen (profesores, padres de familia o agentes de planificación educativa) y desde el lugar en que se hace (práctica educativa, planificación ministerial) [3]. Al resepcto, el Centro Universitario de Desarrollo (CINDA) concluye que calidad en educación superior es un concepto que no existe como tal, sino que es un término de referencia en el cual algo puede ser mejor o peor que otro, dentro de un conjunto de elementos homologables [5]. En consecuencia, en base a las aseveraciones presentadas por la UNESCO y el CINDA, puede afirmarse que una institución, carrera o formación profesional será de 'mejor calidad' que otra cuando presenta criterios homologables y se encuentra con factores contextuales similares.

Otra definición que se atribuye a la calidad de la educación es la centrada en la eficiencia del proceso o del producto educativo, que halla sus orígenes en Franklin Bobbit, quien en 1913 menciona la oportunidad de que el concepto de eficiencia del modo de producción industrial sea aplicable a los problemas de la educación [6].

Tyler [7], en 1949, propone un método racional para encarar, analizar e interpretar el currículo y el sistema de enseñanza de cualquier institución educativa. Se basa en una corriente educativa moderna centrada en los intentos de transformar la educación en un medio eficiente en la formación de ciudadanos y por contraposición a la formación enciclopédica, señalando la dirección que deben tener los esfuerzos para mejorar la calidad de la educación. Este método se centra en establecer ideas de las metas' que persigue la educación. Así, los objetivos curriculares se transforman en eje central de la construcción del currículo, lo que permite guiar las actividades del desarrollo curricular que cambiarán el comportamiento de las personas y, a su vez, se tomarán como criterio para medir la calidad educativa. Del mismo modo, el modelo de excelencia de la Asociación Europea para la Gestión de Calidad (EFQM) menciona que el concepto de calidad es la meta de toda institución educativa, y ésta debe ser funcional, eficaz y eficiente. Funcional, al dar respuesta a la necesidades de formación de la sociedad; eficaz, al lograr las metas educativas de calidad que se había propuesto, y eficiente, al hacer uso correcto de sus recursos. El modelo EFQM presupone que el alcanzar la calidad supone contar con un sistema de gestión de calidad y estar formado en los modelos de evaluación de programas y centros, y sus procedimientos, así como en los modelos de excelencia [8].

El CINDA [9] contempla diversas concepciones de calidad que se han aplicado en la educación superior y además afirma que, con frecuencia, se asevera que el concepto es relativo en varias direcciones; relativo para quien usa el término y las circunstancias en las cuales se invoca. Lo anterior lleva a la conclusión que constituye un término que conlleva los valores del usuario, siendo altamente subjetivo.

En un intento de analizar el concepto, Harvey y Green lo organizaron en cinco criterios: calidad vista como excepción, como perfección, como aptitud para un propósito prefijado, como valor por dinero y como transformación. Estos enfoques se encuentran ligados a la lógica del mercado que prevalece en la mayoría de los países de la región [9].

Otros autores, como Braslavsky [10], han conceptualizado la calidad de la educación como aquella que permite que todos aprendan lo que necesitan aprender, en el momento oportuno de su vida y de sus sociedades y en felicidad.

En consecuencia, toda estrategia para conceptualizar la 'calidad en la educación' deberá priorizar entre una infinidad de componentes involucrados en toda acción educativa, incluyendo factores ambientales, sociales, políticos, económicos, éticos, estructurales, curriculares, administrativos e individuales.

Así, para este estudio se define el concepto de 'educación de calidad' de la siguiente forma: aquel aspecto de la formación profesional que permite a todas las personas aprender lo que necesitan aprender de forma eficiente, con una actitud crítica, en un contexto de pertinencia y responsabilidad personal y social, y que facilita un buen desempeño profesional que les permita construir un sentido profundo y valioso del bienestar humano [10,11].

Para medir la calidad de la formación, resulta necesario evaluar los siguientes criterios que la contienen o que son necesarios para su obtención (Anexo):

- Calidad como recurso [12,13].

- Calidad como proceso $[5,13,14]$.

- Calidad como pertinencia [5,15].

- Calidad como producto (resultado) $[5,12]$.

- Calidad como prestigio [12].

- Calidad como desempeño estudiantil [16,17].

- Calidad como cultura [12,18].

- Calidad como igualdad [19,20]. 
- Calidad como excelencia [12,21].

- Calidad como evaluación [5,21].

Basándose en lo expuesto, el objetivo de la presenta investigación es indagar en la valoración que se realiza de los diferentes criterios del concepto 'calidad en educación’ en la formación del profesional de cinesiología. Se busca conocer y comparar la percepción de estudiantes de último año y de profesionales de primeros años de ejercicio laboral de la carrera de cinesiología. Este conocimiento podría contribuir al análisis amplio del concepto de calidad y, de la misma forma, a la mejora de los procesos educativos y la formación profesional; por consiguiente, a la atención de la salud de la población.

\section{Sujetos y métodos}

\section{Participantes}

La muestra, no probabilística intencionada, estuvo compuesta por 90 participantes mayores de edad de la carrera de cinesiología de la Universidad de Magallanes: 17 estudiantes de quinto año correspondiente al $100 \%$ del curso y 73 profesionales jóvenes egresados. La distribución de los profesionales jóvenes egresados fue de 24 el año 2011, 15 el año 2010, 13 el año 2009, 12 el año 2008 y 9 el año 2007. Un $57,8 \%$ fueron de sexo y un $42,3 \%$ de sexo femenino, con un rango de edad de 23-35 años.

\section{Diseño}

Se utilizó un diseño no experimental transversal correlacional.

\section{Instrumento}

Se confeccionó una herramienta de medición, el cuestionario de percepción de la calidad en la formación profesional del cinesiólogo. Su objetivo es determinar la concepción de los participantes frente al concepto de calidad en la formación del cinesiólogo. Para ello, se elaboraron dos grupos focales: uno para el grupo de jóvenes profesionales y otro para estudiantes de último año. El objetivo de esta fase fue determinar los aspectos generales considerados al referirse a la calidad en la formación. Los resultados obtenidos, junto a lo recogido tras la revisión bibliográfica de la temática, permitieron la elaboración de ítems para el inventario. Dichos ítems se sometieron a una evaluación interjueces expertos con el objetivo de determinar su validez de contenido.
Posteriormente se procedió a una aplicación piloto que permitió evaluar su fiabilidad inicial.

El cuestionario se compone de dos partes. La primera de ellas evalúa el nivel de priorización que los participantes asignan a los criterios antes citados: calidad como recurso, calidad como proceso, calidad como pertinencia, calidad como producto (resultado), calidad como prestigio, calidad como desempeño estudiantil, calidad como cultura, calidad como igualdad, calidad como excelencia y calidad como evaluación. La segunda parte mide la importancia o valor asignado a los criterios a través de un cuestionario consistente en 70 afirmaciones agrupadas en 10 criterios de calidad, que debían evaluarse en su importancia utilizando una escala tipo Likert de 12 puntos (para evitar el punto neutro), desde 1 (poco importante) hasta 12 (indispensable). Estas afirmaciones miden el grado de importancia que asignan los participantes a la calidad como recurso (16 afirmaciones), a la calidad como proceso (16 afirmaciones), a la calidad como pertinencia (6 afirmaciones), a la calidad como producto (5 afirmaciones), a la calidad como prestigio (7 afirmaciones), a la calidad como desempeño estudiantil (4 afirmaciones), a la calidad como cultura (4 afirmaciones), a la calidad como igualdad (4 afirmaciones), a la calidad como excelencia (3 afirmaciones) y a la calidad como evaluación (5 afirmaciones). Puntuaciones elevadas indican una alta importancia en el concepto de calidad en la formación del profesional cinesiólogo. La fiabilidad de este cuestionario alcanzó niveles aceptables ( $\alpha$ de Cronbach $=$ 0,96) (Anexo).

\section{Procedimiento}

Los participantes fueron contactados en sus lugares de estudio y trabajo. Inicialmente se les informó sobre los objetivos generales del estudio y firmaron un consentimiento de participación informada. Luego fueron invitados a responder el cuestionario. Al finalizar la aplicación se contestó a sus preguntas y se les agradeció su participación.

\section{Resultados}

Según se aprecia en la tabla I, las medias totales de importancia atribuidas por los participantes fueron significativamente mayores en los criterios de calidad relacionados con la excelencia, prestigio y producto. Las medias de estos tres criterios considerados como importantes se diferencian significativamente entre sí. En la tabla I también se aprecian 
Tabla I. Promedios de importancia atribuida para cada criterio de calidad evaluada.

\begin{tabular}{lccc}
\hline & Media & $p^{\mathrm{a}}$ & $\mathrm{DE}$ \\
\hline Calidad como recurso & 9,96 & 0,0001 & 1,40 \\
\hline Calidad como proceso & 10,10 & 0,0001 & 1,27 \\
\hline Calidad como pertinencia & 10,25 & 0,0001 & 1,29 \\
\hline Calidad como producto/resultado & 10,35 & 0,0001 & 1,54 \\
\hline Calidad como prestigio & 10,38 & 0,0001 & 1,18 \\
\hline Calidad como desempeño estudiantil & 8,23 & 0,0001 & 2,04 \\
\hline Calidad como cultura & 9,64 & 0,0001 & 1,93 \\
\hline Calidad como igualdad & 9,64 & 0,0001 & 2,07 \\
\hline Calidad como excelencia & 11,04 & 0,0001 & 1,28 \\
\hline Calidad como evaluación & 10,08 & 0,0001 & 1,70 \\
\hline Calidad total & 9,85 & 0,0001 & 1,09 \\
\hline
\end{tabular}

DE: desviación estándar. a Significación en el punto medio $(6,5)$.

los criterios considerados como menos importantes según la media: la calidad como desempeño estudiantil, como cultura y como igualdad. Sin embargo, alcanzan medias superiores al punto medio de la escala, situándose entre las menos significativas al hablar de puntuación total de calidad, pero en términos absolutos se evalúan con alta importancia $\left(\right.$ media $\left.=6,5 ; t_{(90)}=8,04 ; p=0,0001\right)$.

$\mathrm{Al}$ realizar un análisis según rango de importancia, calculado a partir de los resultados totales de calidad -septiles donde 1 (muy bajo) $<8,30$ puntos y 7 (muy alto) > 11,31 puntos-, considerando los porcentajes en que cada criterio fue evaluado como muy indispensable (importancia alta o muy alta), los participantes señalan que la calidad como excelencia es indispensable en la formación profesional del cinesiólogo $(68,9 \%)$, seguida de la calidad como producto $(43,4 \%)$ y de la calidad como prestigio $(41,1 \%)$.

Del mismo modo, al considerar los porcentajes en que cada criterio fue evaluado como poco indispensable (baja o muy baja importancia), las menos señaladas fueron la calidad como cultura $(57,7 \%)$, la calidad como desempeño estudiantil $(56,7 \%)$ y la calidad como igualdad $(27,7 \%)$ (Tabla II).

$\mathrm{Al}$ mismo tiempo que los participantes calificaron la importancia de cada criterio sobre la escala de 12 puntos, se les pidió, en una tarea adicional, organizar estos criterios según el orden de importancia, jerarquizando de más a menos según su opinión.

Los participantes jerarquizan en el primer lugar el criterio de calidad como recurso, otorgándole la mayor importancia. La calidad como producto y la calidad como proceso ocupan el segundo y tercer lugar, respectivamente (Tabla III).

En general, la asignación media de importancia coincide sólo parcialmente con la importancia asignada a cada criterio cuando realizan una tarea de jerarquización.

Al comparar el grado de importancia asignado a cada criterio de calidad en la formación profesional según el grupo al que pertenecen los participantes, en general se puede observar que ambos grupos (profesional joven frente a estudiante de último año) asignan los mismas puntuaciones medias de importancia a los criterios de calidad. Sin embargo, se observan diferencias significativas únicamente para calidad como excelencia y calidad como evaluación. En ambos criterios, los profesionales jóvenes asignan una mayor importancia que los estudiantes de último año. En el caso de excelencia, 11,2 $\pm 1,18$ frente a $10,3 \pm 1,45\left(t_{(90)}=2,64 ; p=0,01\right)$, y de modo similar, en la categoría de calidad como evaluación, los profesionales jóvenes también asignan una mayor importancia a este aspecto que los estudiantes de último año: 10,2 $\pm 1,65$ frente a 9,3 \pm $1,75\left(t_{(90)}=2,00 ; p=0,04\right.$ (Figura).

Al momento de jerarquizar los diferentes aspectos de la calidad según el grupo al que pertenecen los participantes, los profesionales jóvenes establecen que los tres aspectos más importantes son (según porcentaje de elección en las primeras tres posiciones) calidad como recurso (56,1\%), calidad como producto $(49,3 \%$ ) y calidad como proceso $(41,1 \%)$, mientras que los estudiantes de último año señalan calidad como recurso (59,0\%), calidad como evaluación $(58,8 \%)$ y calidad como excelencia $(47,0 \%)$. El aspecto menos importante en la jerarquía según los profesionales jóvenes es calidad como cultura (9,6\%), y según los estudiantes de último año, calidad como pertinencia $(11,8 \%)$ (Tabla III).

\section{Discusión}

Este estudio tuvo como objetivo conocer y comparar la percepción del concepto de calidad en la formación educativa que poseen los profesionales jóvenes y estudiantes de último año de la carrera de cinesiología, e identificar la valoración que asignan a los diferentes aspectos de aquélla. 
Los resultados para el grupo total indican que la calidad como excelencia alcanzó la media más alta. Esto refleja que para los participantes es de gran valor que todos los involucrados se identifiquen con un proyecto colectivo y presenten la adhesión personal a una idea de excelencia en la formación, donde la calidad es responsabilidad de todos y no sólo de aquellos que se encargan de controlarla; esto es compatible con lo mencionado por algunos autores como esencial para conseguir una calidad total $[5,22]$. También llama la atención que la calidad como prestigio y la calidad como producto alcanzan medias altas, lo que puede explicarse por las circunstancias contextuales en las que se encuentran los sujetos del estudio, las cuales están ligadas fuertemente al ingreso en el mercado laboral. Algunos autores afirman que la educación universitaria debe formar estudiantes al servicio del mercado laboral $[23,24]$. Esta tendencia actual de la educación superior podría ejercer una fuerte influencia sobre los resultados obtenidos. Además, podría explicar el bajo promedio de importancia que se otorgó a la calidad como desempeño estudiantil, al ser un criterio ligado a las habilidades interpersonales, académicas y actitudinales frente al proceso de aprendizaje, que en el caso de los profesionales jóvenes constituye una etapa concluida y en el grupo de estudiantes se encuentra en su etapa final.

Otro de los resultados reveló que los profesionales jóvenes asignaron una mayor importancia a los criterios de calidad como excelencia y calidad como evaluación. En consecuencia, este grupo valora la identificación con un proyecto colectivo donde se promuevan valores tales como la consistencia de las 'cosas bien hechas' por parte de los involucrados en la educación, manifestando además que se necesitan eficientes sistemas de evaluación y autoevaluación que velen por el cumplimiento e idoneidad de las estrategias pedagógicas, contenidos teóricos y prácticos, objetivos de los planes de estudio, capacitación docente, desempeño profesional de egresados, etc. que retroalimenten los procesos de formación y contribuyan a mejorar su calidad.

En el análisis de los resultados según el rango de importancia (septil) calculado a partir de los resultados totales, la calidad como excelencia se mantiene en el primer lugar como un elemento indispensable para la formación de calidad. La calidad como producto alcanza el segundo lugar de importancia, desplazando a la calidad como prestigio al tercer lugar en esta categoría. Según este resultado, los sujetos del estudio dan gran importancia al compromiso que deben tener los diferentes actores que participan directamente con la formación de cali-
Figura. Puntuación media de importancia de los criterios de calidad según el grupo estudiado.

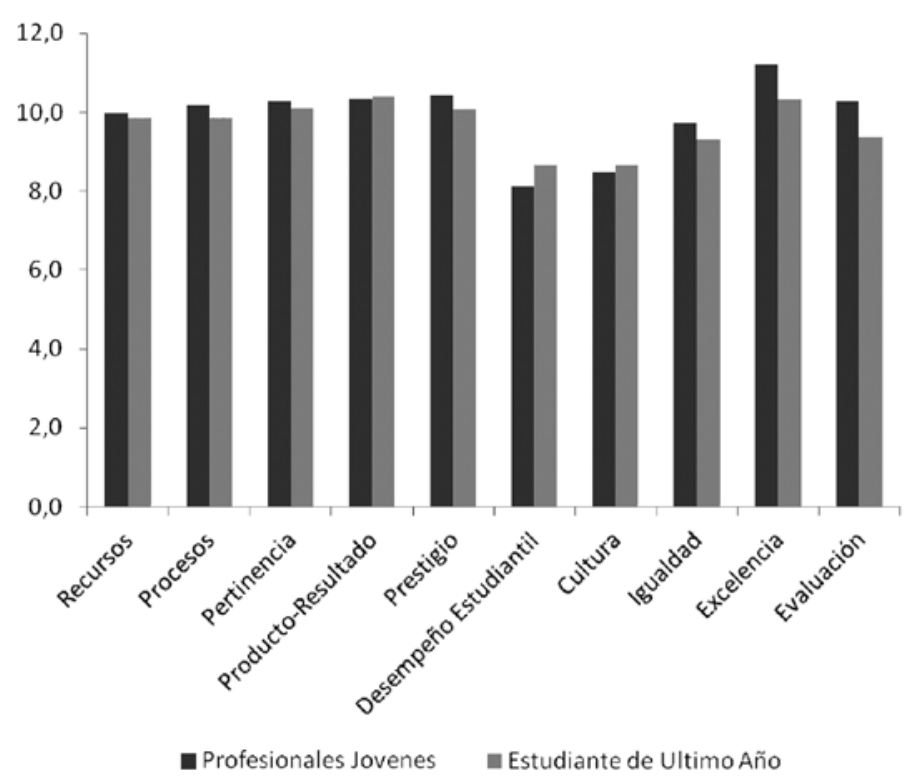

Tabla II. Nivel de importancia asignado a cada criterio de calidad en porcentaje para cada rango cualitativo.

\begin{tabular}{lccccccc}
\hline & Muy baja & Baja & Media baja & Media & Media alta & Alta & Muy alta \\
\hline Recurso & 8,9 & 10,0 & 4,4 & 42,4 & 4,4 & 16,7 & 13,3 \\
\hline Proceso & 8,9 & 7,8 & 5,6 & 36,7 & 5,6 & 18,9 & 16,7 \\
\hline Pertinencia & 7,1 & 9,5 & 3,6 & 33,3 & 6,0 & 11,9 & 28,6 \\
\hline Producto & 11,1 & 2,2 & 1,1 & 34,4 & 7,8 & 17,8 & 25,6 \\
\hline Prestigio & 5,6 & 6,7 & 5,6 & 34,4 & 6,7 & 20,0 & 21,1 \\
\hline $\begin{array}{l}\text { Desempeño } \\
\text { estudiantil }\end{array}$ & 48,9 & 7,8 & 12,2 & 20,0 & 3,3 & 6,7 & 1,1 \\
\hline Cultura & 44,4 & 13,3 & 6,7 & 22,2 & 0 & 6,7 & 6,7 \\
\hline \begin{tabular}{l} 
Igualdad \\
\hline Excelencia
\end{tabular} & 23,3 & 4,4 & 3,3 & 33,3 & 4,4 & 8,9 & 22,2 \\
\hline \begin{tabular}{l} 
Evaluación \\
\hline
\end{tabular} & 12,2 & 10,0 & 2,2 & 32,2 & 5,6 & 12,2 & 25,6 \\
\hline
\end{tabular}

dad, pero ésta debe manifestarse en un resultado positivo como éxito laboral, empleabilidad, evaluación positiva de la organización, comunidad o beneficiarios del servicio profesional, lo que será posi- 
Tabla III. Priorización del grupo total y porcentaje de importancia (primeros tres lugares) según grupo al que pertenecen los participantes.

\begin{tabular}{lccc}
\hline & $\begin{array}{c}\text { Lugar según } \\
\text { jerarquía }\end{array}$ & $\begin{array}{c}\text { Profesionales } \\
\text { jóvenes }\end{array}$ & Estudiantes \\
\hline Recurso & 1 & $56,1 \%$ & $59,0 \%$ \\
\hline Producto & 2 & $49,3 \%$ & $29,4 \%$ \\
\hline Proceso & 3 & $41,1 \%$ & $23,6 \%$ \\
\hline Evaluación & 4 & $39,7 \%$ & $58,8 \%$ \\
\hline Excelencia & 5 & $23,3 \%$ & $47,0 \%$ \\
\hline Prestigio & 5 & $32,9 \%$ & $29,5 \%$ \\
\hline Igualdad & 6 & $27,4 \%$ & $17,7 \%$ \\
\hline $\begin{array}{l}\text { Desempeño } \\
\text { estudiantil }\end{array}$ & 7 & $12,3 \%$ & $41,1 \%$ \\
\hline Pertinencia & 8 & $9,6 \%$ & $11,8 \%$ \\
\hline Cultura & 9 & & $17,7 \%$ \\
\hline & & & \\
\hline
\end{tabular}

ble si la institución, carrera, docentes y estudiantes obtienen resultados favorables en los procesos de acreditación externa, realizan investigación, trabajos comunitarios, etc., que den valor y prestigio social a la institución y a la profesión. Por el contrario, la calidad como cultura es el criterio que presenta menor importancia: los aspectos relacionados con los patrones culturales y sociales son poco valorados por los participantes, lo que puede deberse al carácter profesional y especializado que presenta la formación universitaria en la actualidad, que está al servicio de un mercado laboral cada vez más competitivo y exigente.

Al observar la jerarquización de los 10 criterios de la formación de calidad del grupo total de participantes, éstos consideran que los elementos más importantes son la calidad como recurso, la calidad como producto y la calidad como proceso. El criterio de calidad como cultura resulta el menos relevante. Por consiguiente, para el grupo de estudio es indispensable que una formación de calidad cuente con recursos humanos, infraestructura física, equipamiento y disponibilidad de recursos financieros que den soporte a actividades académicas y sociales, a través de correctos procesos administrativos, organizacionales, curriculares, docentes y pedagógicos que se manifiesten en el éxito laboral del futuro profesional.
Al comparar la jerarquización realizada por ambos grupos (profesionales jóvenes frente a estudiantes de último año), los profesionales jóvenes establecen que los tres aspectos más importantes son calidad como recurso, calidad como producto y calidad como proceso. Sin embargo, el grupo de estudiantes señala que son calidad como recurso, calidad como evaluación y calidad como excelencia, demostrando que este grupo coincide en que los recursos son indispensables, pero además otorga gran valor a que los sistemas de evaluación sean eficientes y con la necesaria identificación con el proyecto de formación profesional por parte de los actores que participan en éste [15]. Todos estos criterios se encuentran fuertemente relacionados con el proceso de formación en el que se halla actualmente el grupo de estudiantes. Este motivo puede influir en que el criterio menos valorado sea el de calidad como pertinencia, que implica estar informado de las políticas del mundo sanitario, del país, del ámbito laboral y cultural [15]. Por el contrario, los profesionales jóvenes dan menos valor al criterio de calidad como cultura, ya que la educación reproduce y construye su cultura y sociedad en correspondencia con la sociedad en que se desarrolla [11].

Una de las limitaciones del presente estudio procede del desequilibrio de la población disponible, ya que el número de estudiantes (18,8\% del total) es bastante menor que el de egresados, lo que es necesario resolver en estudios posteriores para evitar su influencia en los resultados. Además, se recomiendan estudios de tipo longitudinal para conocer en profundidad la variabilidad y evolución del concepto de calidad en educación durante la formación profesional.

En conclusión, este estudio permite determinar los criterios de calidad que son mayoritariamente valorados y consignados como importantes, y evidencia las diferentes percepciones que existen de la calidad educativa entre estudiantes y profesionales, información muy relevante que ayuda a esclarecer un concepto de difícil definición, la 'calidad en educación', que claramente depende de múltiples factores y del contexto de vivencia en el que se encuentra el estudiante o profesional. Además, puede contribuir a la mejora de los procesos educativos en la educación superior (pedagógicos, curriculares, administrativos...), tanto en la formación de pregrado como de posgrado, y por consiguiente, a la formación profesional en general, proporcionando como resultado final la satisfacción de todos los involucrados en la educación. 
Bibliografía

1. Comisión Nacional de Acreditación de Pregrado. Chile. Sistema Nacional de Aseguramiento de la Calidad de Educación Superior. URL: http://www.cnachile.cl/wp-content/uploads/ 2010/06/Ley_Aseg_Calidad.pdf. [30.01.2013].

2. De la Orden A, Asensio I, Carballo R, Fernández-Díaz J, Fuentes A, García-Ramos JM, et al. Desarrollo y validación de un modelo de calidad universitaria como base para su evaluación. Revista Electrónica de Investigación y Evaluación Educativa 1997. URL: http://www.uv.es/RELIEVE/v3n1/ RELIEVEv3n1_2.htm. [30.04.2012].

3. Edwards-Risopatrón V. El concepto de calidad de la educación. Santiago de Chile: UNESCO/OREALC; 1991.

4. Zurita-Chávez, R. El problema de la calidad de la educación aproximación a un concepto. Temuco, Chile: Universidad de la Frontera/PREDE/OEA; 1987.

5. González E, Ayarza E. Calidad, evaluación institucional y acreditación en la educación superior en la región Latinoamericana y del Caribe. URL: http://www.una.edu.ni/otei/ documentos/Autoevaluacion/Autoevaluacion\%202012/ calidad-evaluacion-y-acreditacion.pdf. [20.12.2012]

6. Bobbit $F$. The supervision in city schools: some general principles of management applied to the problems of city-school systems. Bloomington, 12th: Yearbook of the National Society for the Study of Education 1913; II: 11-96.

7. Tyler R. Principios básicos de currículo. Buenos Aires: Troque; 1949.

8. Martínez-Mediano C. El modelo de excelencia en la EFQM y su aplicación para la mejora de la calidad de los centros educativos. Educación XXI 2005; 8: 35-65.

9. Espinoza O, González F, Poblete A, Ramírez S, Silva T, Zúñiga M. Autoevaluación para instituciones de educación superior, pautas y procedimientos. Santiago de Chile: CINDA/PROMESUP-OEA; 1994.

10. Braslavsky C. Diez factores para una educación de calidad para todos en el siglo XXI. REICE 2006; 4: 84-101.

11. Salas-Perea R. La calidad en el desarrollo profesional: avances y desafíos. Educ Med Super 2000; 14: 136-47.

12. Duque-Oliva E, Chaparro-Pinzón C. Medición de la percepción de la calidad del servicio de educación por parte de los estudiantes de la UPTC Duitama. Criterio Libre 2012; 10: 159-92.
13. Alterio-Ariola G, Pérez-Loyo H. Evaluación de la función docente según el desempeño de los profesores y la opinión estudiantil. Educ Med Super 2009; 23: 1-14.

14. Aguerrondo-Braslavsky I. La calidad de la educación: ejes para su definición y evaluación. La Educación 1993; 116: 561-78.

15. Pérez-Juste R. Calidad de la educación, calidad en la educación. Hacia su necesaria integración. Educación XXI 2005; 8: 11-33.

16. González-López I. Dimensiones de la evaluación de la calidad universitaria en el Espacio Europeo de Educación Superior. Revista Electrónica de Investigación Psicoeducativa 2006. URL: http://redalyc.uaemex.mx/src/inicio/ArtPdfRed. jsp?iCve=293121929001. [05.12.2012].

17. Flórez-Peña $\mathrm{T}$, Rojas-Cristancho J. Factores asociados a la calidad en las facultades de derecho de alto rendimiento académico en Colombia. Educación y Educadores 2010; 13: 185-97.

18. Nuviala-Nuviala A, Casajús-Mallén J. Calidad percibida del servicio deportivo en edad escolar desde la perspectiva de los padres. El caso de la provincia de Huelva. Revista Internacional de Medicina y Ciencias de la Actividad Física y del Deporte 2005; 5: 1-12.

19. UNESCO. Diez aspectos de la educación para todos. URL: http://www.unesco.org/es/efa/the-efa-movement/ 10-things-to-know-about-efa/. [03.12.2012].

20. UNESCO. Una educación de calidad para todos los jóvenes. 47. ${ }^{a}$ Conferencia Internacional de Educación de la UNESCO. Ginebra, 8-11 de Septiembre de 2004. URL: http://www.ibe. unesco.org/fileadmin/user_upload/archive/publications/ free_publications/educ_qualite_esp.pdf. [03.12.2012].

21. Sanyal B, Martin M. Garantía de la calidad y el papel de la acreditación: una visión global. URL: http//upcommons.upc. edu/revistes/bitstream/2099/7499/1/02_3-19.pdf. [14.12.2012].

22. López-Rupérez F. Gestión de calidad en educación. 3 ed. Madrid: La Muralla; 2003.

23. Layer, G. Widening participation and employability. York: Learning and Teaching Support Network; 2004.

24. Semeijn JH, Van der Velden R, Heijke H, Van der Vleuten C, Boshuizen HPA. Competence indicators in academic education and early labour market success of graduates in health sciences. J Educ Work 2006; 19: 383-413.

\section{Anexo. Encuesta de percepción del concepto de calidad en la formación profesional del cinesiólogo}

\section{Instrucciones}

A continuación se presentan 10 definiciones de conceptos que se identifican con la formación de calidad de un cinesiólogo. Debes priorizar los conceptos, asignando el valor 10 a la definición de menor importancia para la formación de calidad y así sucesivamente, hasta asignar el valor 1 al concepto indispensable para la educación de calidad. Cada número puede ser asignado sólo una vez:

Calidad en recursos. Se entenderá por calidad en recursos la cantidad y características de los recursos humanos, infraestructura física, equipamiento y disponibilidad de recursos financieros, que dan soporte a actividades académicas y sociales desarolladas por los estudiantes.
Calidad como procesos. Se refiere a la calidad producto de la interacción de diversos elementos y fuerzas que intervienen en la marcha y desarrollo de institución. En este ítem se incluyen aspectos administrativos, organizativos, curriculares, docentes y pedagógicos.

Calidad como pertinencia. Se refiere a la calidad entendida como la adecuada sincronización de la formación profesional con el contexto, país, región, grupo social y profesión.

Calidad como producto (resultado). Calidad de la formación que se manifiesta en el éxito laboral entendido como empleabilidad y también como evaluación positiva de la organización o el paciente que recibe el servicio profesional.

Calidad como prestigio. Calidad entendida como 
la evaluación prestigiosa tanto de la institución que la imparte como de la carrera específica que ejecuta la formación profesional. Contempla elementos como la investigación, el trabajo comunitario, resultados favorables en procesos de acreditación de escala nacional, etc., y su valoración en el ámbito comunitario.

Calidad como desempeño estudiantil. Se entenderá como la calidad que depende de las habilidades interpersonales y académicas que poseen de base los estudiantes, como también su actitud frente al proceso de aprendizaje y su capacidad para transformarse como persona.

Calidad como cultura. Es aquel aspecto de la calidad que se vincula con una formación profesional que incluya los patrones culturales y los valores de la sociedad, incluyendo al propio individuo y su rol social, y como éste entiende desde su subjetividad la calidad.

Calidad como igualdad. Es aquel aspecto de la calidad que consiste en ofrecer una igualdad efectiva de oportunidades educativas e igualdad en los procesos de aprendizaje a todos los estudiantes, sin excepciones a través de una educación permanente y sin fronteras, donde el mérito sea el criterio básico para el acceso y se atiendan también aspectos individuales o particulares asociados a la formación.

Calidad como excelencia. Se refiere a la calidad asociada al proceso de formación profesional en el que se promueven valores tales como la consistencia de las 'cosas bien hechas' que van más allá de los requisitos legales o técnicos.

Calidad como evaluación. Aspecto de la calidad que se vincula con la eficacia de los sistemas de evaluación durante la formación, y que se constituyen en un aspecto fundamental de la misma en forma de retroalimentación. Se asocia además con la orientación a autoevaluarse, reconociendo carencias y debilidades, e identificando recursos que permitan afrontar adecuadamente su resolución.

A continuación se presentan una serie de preguntas acerca de la formación profesional del cinesiólogo. Te solicito leer atentamente y responder en el cuestionario el valor o importancia (de 1 a 12) que otorgas a cada aspecto de la formación, considerando que 1 equivale a 'poco importante', y 12, a 'indispensable':

\section{Calidad como recurso}

1. Al evaluar la calidad de la educación es fundamental considerar que la carrera cuente con dispositivos audiovisuales.

2. Al evaluar la calidad educativa es fundamental considerar las condiciones de las salas de clases.
3. El mobiliario de las salas de clases es un elemento primordial al medir la calidad de la educación.

4. El número de salas de clases es un elemento fundamental a la hora de evaluar la calidad educativa.

5. Condiciones ambientales, como la dimensión de la sala de clases, son un elemento relevante al evaluar la calidad de la educación.

6. La disponibilidad de salas de clases es un elemento primordial a la hora de evaluar la calidad de la educación.

7. Contar con espacios físicos para estudiar es un elemento relevante al evaluar la calidad de la educación.

8. Los equipos de laboratorios con los que cuenta una carrera son fundamentales para garantizar una educación de calidad.

9. La cantidad de material que poseen los laboratorios por estudiante es un elemento relevante a la hora de evaluar la calidad de la educación.

10. Al evaluar la calidad educativa es muy importante considerar la disponibilidad de insumos de laboratorio.

11. El buen estado de los equipos de laboratorio es fundamental al evaluar la calidad educativa.

12. Contar con materiales propios de la formación cinésica es fundamental para garantizar una educación de calidad.

13. Contar con recursos financieros de libre disposición para que las carreras realicen actividades académicas (jornadas, simposios, etc.) es fundamental para garantizar una educación de calidad.

14. Una educación de calidad requiere necesariamente un número adecuado de docentes para una educación de calidad.

15. Al evaluar calidad de la educación es fundamental que los docentes ejerzan activamente la especialidad en la cual desempeñan labores docentes.

16. La cantidad de recursos bibliográficos que posee una carrera es fundamental para garantizar una educación de calidad.

\section{Calidad como proceso}

1. Una educación de calidad considera necesariamente que se expliciten los principios que fundamentan la organización de un programa de estudios.

2. Una educación de calidad considera necesariamente que los procesos administrativos (organización horaria, informaciones académicas, distribución de salas, etc.) estén estructurados para facilitar la docencia.

3. La existencia de normas o procedimientos que regulan el quehacer administrativo es un elemento muy importante a considerar al medir la calidad educativa. 
4. Un elemento importante a considerar al evaluar calidad educativa es el cumplimiento de las normas que rigen los procesos académicos.

5. La existencia de canales de comunicación expeditos entre docentes, administrativos y estudiantes es un elemento muy importante a considerar al medir la calidad educativa.

6. El seguimiento y cumplimiento del diseño curricular es un aspecto fundamental al evaluar la calidad de la educación.

7. Una educación de calidad requiere que el programa de estudios se organice de manera secuencial.

8. Una educación de calidad requiere que las asignaturas estén organizadas secuencialmente.

9. El cumplimiento del plan de estudio es un aspecto fundamental al evaluar la calidad de la educación.

10. El cumplimiento de los programas de estudio es un aspecto fundamental al evaluar la calidad de la educación

11. Una educación de calidad considera necesariamente que los programas de las asignaturas estén actualizados.

12. Las metodologías de enseñanza son muy relevantes al medir la calidad de la educación.

13. La evaluación de las estrategias metodológicas y didácticas que utiliza un docente es un elemento muy importante a considerar cuando se evalúa la calidad educativa.

14. La disponibilidad horaria del docente, fuera del tiempo de clase, es un elemento muy importante a considerar al medir la calidad educativa.

15. Al evaluar la calidad de la educación es importante considerar que los docentes cuenten con despacho y horario de atención a estudiantes.

16. Un elemento importante a considerar al evaluar la calidad educativa es que los docentes reciban sanciones al ser mal evaluados.

\section{Calidad como pertinencia}

1. Al evaluar la calidad de la educación considera importante que las carreras satisfagan las necesidades de la sociedad o comunidad en la cual se encuentran insertas.

2. Que los egresados de la carrera resuelvan problemas de la comunidad o sociedad, del ámbito local, es un criterio relevante al evaluar la calidad educativa.

3. La valoración comunitaria de una carrera es un elemento importante a considerar cuando se evalúa la calidad de la educación.

4. Al evaluar la calidad de la educación considera importante que las carreras satisfagan las necesidades laborales del mercado.
5. Que la carrera se involucre con fundaciones o sociedades de personas con discapacidad es un elemento importante a considerar en una educación de calidad.

6. Que la formación de la carrera se encuentre alineada con los objetivos sanitarios del país es un aspecto importante en una educación de calidad.

\section{Calidad como producto (resultado)}

1. El reconocimiento profesional de los egresados de una carrera, por parte de la comunidad, es un elemento importante a considerar cuando se evalúa la calidad educativa.

2. El reconocimiento profesional de los egresados de una carrera, por parte de la organización en la que trabaja, es un elemento importante a considerar cuando se evalúa la calidad educativa.

3. El reconocimiento profesional de los egresados de una carrera, por parte de sus pacientes, es un elemento importante a considerar cuando se evalúa la calidad educativa.

4. La empleabilidad de los egresados de una carrera es un elemento importante a considerar cuando se evalúa la calidad de la educación entregada.

5. Que los egresados de la carrera de cinesiología de la Universidad de Magallanes sean contratados en servicios públicos, por encima de profesionales procedentes de otras instituciones, es un aspecto importante a considerar cuando se evalúa la calidad educativa.

\section{Calidad como prestigio}

1. Al evaluar la calidad de la educación es importante considerar que la carrera sea reconocida internacionalmente.

2. Al evaluar la calidad de la educación es importante considerar que la universidad cuente con acreditación nacional.

3. Que la carrera se encuentre acreditada en el ámbito nacional es un elemento importante a considerar para evaluar la calidad de la educación.

4. Las investigaciones y publicaciones que posee la carrera constituyen un elemento de prestigio importante a considerar en una educación de calidad.

5. El trabajo comunitario que realiza una carrera es un elemento de prestigio importante a considerar en una educación de calidad.

6. Que la formación profesional otorgada en la carrera sea bien valorada por otros cinesiólogos es un elemento importante a considerar para evaluar la calidad de la educación.

7. Que la formación profesional otorgada en la carrera sea bien valorada por otros profesionales 
de la salud es un elemento importante a considerar para evaluar la calidad de la educación.

\section{Calidad como desempeño estudiantil}

1. El rendimiento académico de los estudiantes durante su proceso de formación es un elemento importante a considerar al evaluar la calidad educativa.

2. La puntuación en la prueba de selección universitaria que tiene un estudiante es un elemento muy importante a considerar cuando se evalúa la calidad educativa.

3. La formación académica que posee un estudiante, previamente a su ingreso en la universidad, es un elemento importante al evaluar la calidad educativa

4. Las habilidades de aprendizaje que posean los estudiantes durante su proceso de formación profesional constituyen un elemento muy importante a considerar cuando se evalúa la calidad educativa.

\section{Calidad como cultura}

1. Que una carrera o institución considere los patrones culturales de la población, durante la formación profesional, es un elemento fundamental para conseguir una educación de calidad.

2. Que una carrera profesional considere en su formación potenciar a la persona en su rol social es un elemento importante para conseguir una educación de calidad.

3. Que la carrera considere que, a través del acceso a la educación superior, el estudiante mejorará su condición económica es un elemento importante cuando se evalúa la calidad de la educación.

4. Para alcanzar una educación de calidad es necesario que una carrera profesional enseñe a sus estudiantes la cultura y los valores de la sociedad en la que se encuentra.

\section{Calidad como igualdad}

1. Un elemento importante a considerar al evaluar la calidad educativa es el ingreso igualitario a una carrera (estudiar la profesión debe ser posible para todos los estudiantes).

2. Un elemento importante a considerar al evaluar la calidad educativa es que la formación debe ser igual para todos.

3. Al evaluar la calidad educativa es importante considerar la existencia de programas de nivelación para los estudiantes.

4. Al evaluar la calidad educativa es importante considerar que el programa de estudios respete la individualidad de sus estudiantes.

\section{Calidad como excelencia}

1. Que el docente tenga el deseo de realizar bien sus funciones es un aspecto fundamental a considerar en una educación de calidad.

2. Que el estudiante posea la disposición de realizar bien sus funciones académicas es un aspecto importante a considerar en una educación de calidad.

3. Para alcanzar una educación de calidad es necesario que toda la organización realice sus funciones de forma correcta.

\section{Calidad como evaluación}

1. Los sistemas de autoevaluación que posea un programa de estudios son relevantes al medir la calidad educativa.

2. Los sistemas de evaluación docentes que posea un programa de estudios son relevantes al medir la calidad educativa.

3. Los sistemas de evaluación para el cumplimiento de los objetivos de las asignaturas son relevantes al medir la calidad educativa.

4. La validación de las evaluaciones es un elemento muy importante a considerar cuando se evalúa la calidad educativa.

5. La evaluación del desempeño profesional de los egresados es un elemento muy importante a considerar cuando se evalúa la calidad educativa. 